\title{
Outer stellar disks and star-forming rings in S0 galaxies
}

\author{
I. S. Proshina ${ }^{1}$, A. Yu. Kniazev ${ }^{1,2}$ and O. K. Sil'chenko ${ }^{1}$ \\ ${ }^{1}$ Sternberg Astronomical Institute, Lomonosov Moscow State University, \\ University av. 13, 119991 Moscow, Russia \\ email: ii.pro@mail.ru, olga@sai.msu.su \\ ${ }^{2}$ South African Astronomical Observatory and SALT Foundation, \\ PO Box 9, 7935 Observatory, Cape Town, South Africa \\ email: akniazev@saao.ac.za
}

\begin{abstract}
We have studied a small sample of star-forming outer rings in unbarred lenticular galaxies by means of long-slit spectroscopy. We have determined metallicities of the outer stellar disks and of the ionized gas in the rings. While the stellar disks of the S0s are metal-poor, $[\mathrm{Z} / \mathrm{H}]<-0.4$, the gas has strictly solar oxygen abundance in all the cases.
\end{abstract}

Keywords. galaxies: elliptical and lenticular, cD - galaxies: evolution - galaxies: formation

S0 galaxies are disk galaxies which differ from spirals by smooth appearance of their reddish large-scale stellar disks. They belong to red sequence and are often characterized as 'quiescent' galaxies. However, the most S0s possess cold gas, and sometimes they reveal low-level star formation organized in rings. Here we study a few S0 galaxies where outer UV-bright rings are present. In NGC 809 and NGC 7808 the UV-rings were reported by the GALEX team (Gil de Paz et al. 2007); for NGC 4324 the UV-ring is presented by Cortese \& Hughes (2009). PGC 48114 was noted as a ring galaxy by Kostyuk (1975), and our inspection of its GALEX image has confirmed the UV-ring in this galaxy.

The long-slit spectroscopy was made with the Robert Stobie Spectrograph (RSS) at the $11 \mathrm{~m}$ Southern African Large Telescope (SALT) by using a $1.25^{\prime \prime}$ slit width. The volume-phase grating PG0900 covered the spectral range of 3760-6860 $\AA$ with a final reciprocal dispersion of about $0.97 \AA$ per pixel and FWHM spectral resolution of $5.5 \AA$.

After deriving long-slit spectra of the galaxies, we have measured the equivalent widths of the emission lines detected within the rings and Lick indices of the stellar populations in the vicinity of the rings. The stellar population SSP-equivalent ages and metallicities were determined by confronting the observed Lick indices to the models by Thomas et al. (2003). While the stellar ages are various in the outer disks of the galaxies, ranging from 1.5 Gyr in NGC 2697 to 12 Gyr in NGC 4324, the stellar metallicity is everywhere low, $[\mathrm{Z} / \mathrm{H}]<-0.4$. The gas in the rings is excited mostly by young massive stars in 4 of 5 galaxies. We have estimated the metallicity of the gas by applying the calibration of the [NII] to $\mathrm{H} \alpha$ ratio from Pettini \& Pagel (2004). We have found that the warm gas of the star-forming rings has solar O-abundance in all 4 galaxies where it was measured.

\section{References}

Cortese, L. \& Hughes, T. M. 2009, MNRAS, 400, 1225

Gil de Paz, A., et al. 2007, ApJS, 173, 185

Kostyuk, I. P. 1975, Soobshch. Spets. Astrofiz. Obs. 13, 45

Pettini, M. \& Pagel, B. E. J. 2004, MNRAS, 348, L59

Thomas, D., Maraston, C., \& Bender, R. 2003, MNRAS, 339, 897 